\title{
Estudio de prácticas administrativas financieras en PYMES de Riobamba y su impacto en la gestión
}

Study of financial administrative practices in smes of Riobamba and its impact on management

Angelita Genoveva Tapia Bonifaz. ${ }^{1}$, Sandra Patricia Jácome Tamayo. ${ }^{2}$, María Isabel Gavilánez Vega. ${ }^{3}$ \& Jaqueline Elizabeth Balseca Castro. ${ }^{4}$

\begin{abstract}
DOI: https://doi.org/10.33262/cienciadigital.v3i3.2.720

This study aims to determine the financial administrative practices that are applied in the SMEs of the city of Riobamba and their impact on management indicators, for which 60 managers were surveyed, which allowed identifying which of the companies surveyed at least $52 \%$ perform an elementary management for the development of their business activities. When asking the managers, if after a fiscal year, in their companies perform a situational analysis of their administrative and financial structure, which allows decisions to be made based on precise data, $75 \%$ answered affirmatively. Considering that planning is the basis of the administration, it was asked if the actions developed in their organizations are carried out prior to planning, 58\% of the respondents state that their actions are arranged according to a schedule prepared by the managers. Finally, $77 \%$ of the surveyed businessmen consider that the development of a financial administrative manual will be a tool that will contribute to an effective administrative and financial management that favors the sustainability and sustainability of the companies.
\end{abstract}

\footnotetext{
${ }^{1}$ Escuela Superior Politécnica de Chimborazo, Facultad de Administración de Empresas, Riobamba, Ecuador, genoveva.tapia@espoch.edu.ec

2 Escuela Superior Politécnica de Chimborazo, Facultad de Administración de Empresas, Riobamba, Ecuador, sandra.jacome@espoch.edu.ec

${ }^{3}$ Escuela Superior Politécnica de Chimborazo, Facultad de Administración de Empresas, Riobamba, Ecuador, maria.gavilanez@espoch.edu.ec

${ }^{4}$ Escuela Superior Politécnica de Chimborazo, Facultad de Administración de Empresas, Riobamba, Ecuador, jaquelinebalseca@yahoo.es
} 
Keywords: Financial Administrative Practices, SMEs, Management Indicators

\section{RESUMEN}

El presente estudio pretende determinar las prácticas administrativas financieras que se aplican en las Pymes de la ciudad de Riobamba y su impacto en los indicadores de gestión, para lo cual se encuestó a 60 administradores, de las mismas, lo que permitió identificar que de las empresas encuestadas al menos el 52\% realizan una gestión elemental para el desarrollo de sus actividades empresariales. Al preguntarles a los directivos, si luego de un ejercicio fiscal, en sus empresas efectúan un análisis situacional de su estructura administrativa y financiera, que permita tomar decisiones en base a datos precisos, el $75 \%$ respondieron afirmativamente. Considerando que la planificación es la base de la administración se preguntó si las acciones desarrolladas en sus organizaciones se realizan previo a una planificación, el 58\% de los encuestados manifiestan que sus acciones están dispuestas de acuerdo a una programación elaborada por parte de los directivos. Finalmente, el $77 \%$ de los empresarios encuestados, consideran que el desarrollo de un manual administrativo financiero será una herramienta que contribuirá a una efectiva gestión administrativa y financiera que favorezca la sustentabilidad y sostenibilidad de las empresas.

Palabras claves: Prácticas Administrativas Financieras, PYMES, Indicadores de Gestión

\section{Introducción}

La Integración de los mercados a través del avance tecnológico y los medios de comunicación cada vez más avanzados, requiere que todas las organizaciones grandes o pequeñas sean dinámicas acorde a los nuevos cambios y tendencias. La mayor parte de las Pymes son empresas familiares que por su estructura organizacional no han tenido un desarrollo formal, han sido administradas de manera empírica, no poseen sistemas de información financiera que permitan la toma de decisiones adecuadas y oportunas, en consecuencia, se ha detenido su crecimiento y competitividad.

Por lo expuesto, se ha realizado un estudio que permita conocer las prácticas administrativas y financieras, que se aplican en las Pymes de Riobamba, con el fin de analizarlas y proponer estrategias para mejorar la gestión que realizan este tipo de organizaciones, que en la actualidad se han convertido en generadoras de fuentes de empleo y constituyen un factor dinamizador de la economía de la ciudad.

Se ha considerado como punto de partida, una base teórica que permita dar sustento técnico a la exploración de conocimientos, con proposiciones probadas, que orienten el trabajo de investigación y a la vez permitan emitir propuestas que se adapten a las necesidades 
administrativas y financieras de las Pymes.

La investigación tiene un enfoque cuali- cuantitativo, en la metodología empleada se aplicaron encuestas a los administradores de 60 empresas, para recabar información que evidencie el uso de las prácticas administrativas y financieras que utilizan para alcanzar la eficacia y eficiencia en el desarrollo de sus actividades; lo cual permite realizar un análisis de su gestión administrativa y financiera.

El esquema de lineamientos variados que se examina en las Pymes en estudio, fue cualitativo porque se consideran las opiniones vertidas por los empresarios en las encuestas ejecutadas y analizadas, así como también, es un estudio cuantitativo por que se determina en forma numérica los resultados del estado actual de las prácticas administrativas y financieras, valuando los diferentes matices que se presentan en las organizaciones; lo que permite concluir que hay la necesidad de aplicar un manual que se adapte a cualquier tipo de Pymes.

\section{Fundamentación Teórica}

\section{Definición de PYME}

(La Comisión de la Comunidad Andina, 2008), en la decisión 702 determina que la PYME comprende a todas las empresas formales legalmente constituidas y/o registradas ante las autoridades competentes, que lleven registros contables y/o aporten a la seguridad social, comprendidas en el Artículo 3. No se incluyen a las Unidades Productivas Informales (UPI).

Para fines tributarios las PYMES de acuerdo al tipo de RUC que poseen se las divide en personas naturales y sociedades.

\section{Importancia de las PYME}

(Martínez, 2016), no es aventurado decir que las PYMES son el motor más importante del desarrollo de cualquier país, tienen una mayor capacidad de ampliación y de generación de nuevos empleos y por tanto son las que más pueden contribuir al crecimiento del PIB.

Para (Luna, 2013), la importancia de la pequeña y mediana empresa reside en la capacidad para ofrecer empleo, en su flexibilidad para aumentar la oferta de satisfactores y en su habilidad para adaptarse a regiones que es necesario promover dentro de un programa que tome en cuenta de desarrollo económico equilibrado.

Para el desarrollo de la provincia las Pymes son esenciales, han contribuido a dinamizar la economía Chimboracense, considerando que son generadoras de nuevas oportunidades de empleo para la gran cantidad de jóvenes profesionales de distintas especialidades, que surgen de tres prestigiosas universidades. 


\section{Gestión y su importancia}

Para (Beltrán Jaramillo, 2000), la gestión es la acción de administrar, es decir, hacer diligencias conducentes al logro de un negocio; coloca al empresario frente a un estilo gerencial.

Acción y efecto de administrar; esto es equivalente a gestionar, que es asignar recursos, controlar y evaluar su utilización de acuerdo con el cumplimiento de objetivos y siguiendo estrategias y políticas que pueden no estar cuantificadas, ni explicitadas. (Greco, 2003, p.225)

La producción es el corazón de la industria, por eso ejercer la correcta administración de todos los recursos en forma organizada, planificada y controlada permitirá generar la riqueza que se convierte en la meta de las entidades.

\section{Dinámica de la Gestión}

La dinámica es una integración sistemática que permite llegar al cumplimiento de un objetivo considerando como base sus diferentes etapas, como se observa en la figura 1.

\section{El Control de Gestión}

El Control de Gestión es un sistema de información estadística, financiera, administrativa y operativa que puesta al servicio de la directiva de la organización, le permite tomar decisiones acertadas y oportunas, adoptar medidas correctivas que correspondan y controlar la evolución en el tiempo de las principales variables y procesos (Beltrán Jaramillo, 2000).

Esto se podría encontrar si las organizaciones son eficientes en la toma de decisiones exhortando estándares establecidos y que logren ser competitivas en todos los mercados globalizados.

Figura. 1 Dinámica de la Gestión

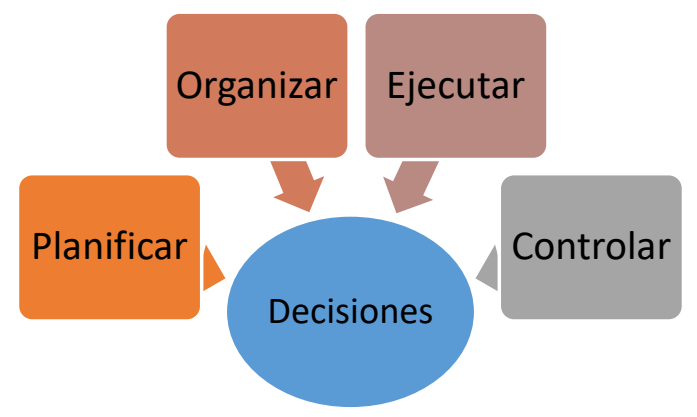

Fuente: (Pérez \& Veiga, 2013)

Elaborado por: Autores

\section{Elementos del Control de Gestión}

Según Muñiz (2004) los elementos del sistema de control de gestión son: herramientas y soportes, sistema de costes, control presupuestario y el sistema de información. 
Las herramientas y soportes son: el plan de cuentas, la codificación analítica y el sistema de control interno; el programa de gestión informática integrado; soporte del departamento de informática; conocimientos de los riesgos fiscales; el benchmarking.

El sistema de costes determinará el origen, calidad, control y riesgos.

El control presupuestario requiere de una planificación, evaluación y control.

En el sistema de información es importante, establecer indicadores que permitan evaluar el cumplimiento de los objetivos para la toma de decisiones oportunas.

Un indicador es una herramienta que nos permite valorar con certeza, tanto cuantitativamente como cualitativamente, el desempeño de los procesos, con el fin de identificar desviaciones y tomar acciones correctivas que aporten de forma axiomática a la mejora continua.

Por consiguiente, se podría decir que el control de la gestión en cualquier organización es imprescindible ya que nos permite medir y verificar la eficiencia con la que se están desarrollando las diferentes fases y tomar los correctivos necesarios de ser el caso en forma oportuna.

\section{Administración}

Desde el punto de vista de (Stoner, Freeman, \& Gilbert, 1996) la administración es el proceso de planificar, organizar, dirigir y controlar las actividades de los miembros de la organización y el empleo de todos los demás recursos organizacionales, con el propósito de alcanzar las metas establecidas para la organización.

\section{Gestión Administrativa}

La gestión administrativa consiste en todas las actividades que se emprenden para coordinar el esfuerzo de un grupo, es decir la manera en la cual se tratan de alcanzar las metas u objetivos con ayuda de las personas y las cosas mediante el desempeño de ciertas labores esenciales como son la planeación, organización, dirección y control. (Anzola Rojas, 2002).

La gestión financiera, se considera como una ciencia que fomenta el correcto uso del dinero y como negociar el mismo para mejorar su valor.

"La gestión financiera es el área de la administración que tiene que ver con los recursos financieros de la empresa y se centra en dos aspectos importantes como son, la rentabilidad y la liquidez.” (Córdoba, 2012, p.7)

Toda organización requiere de recursos económicos para el desarrollo de sus objetivos, por ello es importante que las decisiones sobre la obtención y la asignación de fondos financieros sea racional para cubrir las necesidades de la entidad. 
Por lo expuesto, la gestión financiera de una empresa es una de las claves, que permiten reflejar en términos pecuniarios las inversiones previstas, puesto que, se realiza con previsión, los egresos e ingresos que se van a efectuar en la organización y la posible rentabilidad que se espera; así como su viabilidad financiera en un tiempo determinado.

\section{Planificación}

La planificación podríamos expresar como un sistema estratégico continuo con la disposición de actividades de acuerdo a los tiempos establecidos por cada organización, considerando las diferentes variables sociales, económicas y técnicas para la toma de decisiones, que le permitan alcanzar el máximo objetivo, con el menor esfuerzo y al menor costo posible. (Tapia Bonifaz, 2014)

\section{Matriz FODA}

La matriz FODA es un instrumento de análisis conocido también como análisis SWOT por sus siglas en inglés. Las letras de la palabra FODA significan fortalezas, oportunidades, debilidades y amenazas.

Las fortalezas y debilidades corresponden al análisis del ambiente interno; mientras que las amenazas o riesgos y las oportunidades al ámbito externo, como se observa en la figura 2. (Cipriano, 2016).

\section{Figura. 2 Matriz FODA}

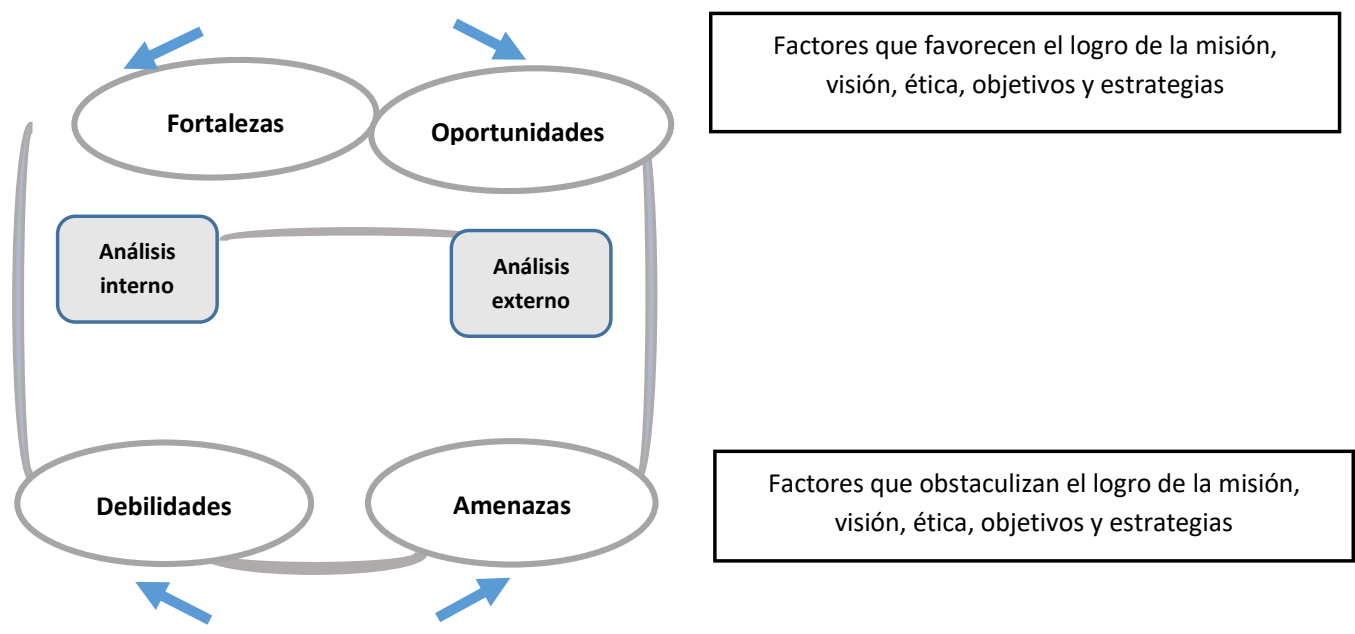

Fuente: (Cipriano, 2016)

\section{Elaborado por: Autores}

Por consiguiente, esta herramienta administrativa permite conseguir información real y actualizada de la organización en estudio en un periodo determinado, con el fin de implementar estrategias encaminadas al logro de los objetivos empresariales. 


\section{Metodologia.}

Con la finalidad de recolectar información que permita analizar las prácticas administrativas y financieras de las PYMES de Riobamba, se inicia con una investigación exploratoria, donde se examinan escenarios que permiten recopilar información efectiva para realizar un análisis cualitativo considerando las opiniones vertidas por los empresarios entrevistados, lo que permite establecer las preguntas pertinentes para realizar un estudio cuantitativo por medio de valoraciones numéricas de los resultados del estado actual de las prácticas administrativas y financieras, valuando diferentes matices que se presentan en las organizaciones, para lo cual se consideró una muestra de 60 PYMES

\section{Resultados}

\subsection{Elaboración de la Matriz FODA}

Se realiza una matriz FODA de las Pymes de Riobamba que permita determinar la situación actual de la gestión administrativa y financiera, tal como se muestra en la Tabla $\mathrm{N}^{\circ} 1$

Tabla 1. Matriz FODA Pymes Riobamba

\begin{tabular}{|c|c|}
\hline \multicolumn{2}{|c|}{ Factores Internos } \\
\hline Fortalezas & Debilidades \\
\hline & $\begin{array}{l}\text { Estructura } \\
\text { organizacional no }\end{array}$ \\
\hline Precios competitivos & definida \\
\hline $\begin{array}{l}\text { Experiencia en el } \\
\text { mercado }\end{array}$ & $\begin{array}{l}\text { Insuficientes recursos } \\
\text { económicos } \\
\text { Sistema de control de }\end{array}$ \\
\hline Guías de planificación & $\begin{array}{l}\text { interno } \\
\text { Volúmenes de }\end{array}$ \\
\hline Personal capacitado & producción \\
\hline $\begin{array}{l}\text { Prestigio en el } \\
\text { mercado }\end{array}$ & $\begin{array}{l}\text { Sistema de control de } \\
\text { calidad }\end{array}$ \\
\hline $\begin{array}{l}\text { Diversificación de } \\
\text { Productos }\end{array}$ & \\
\hline \multicolumn{2}{|c|}{ Factores Externos } \\
\hline Oportunidades & Amenazas \\
\hline Alianzas estratégicas & \\
\hline de comercialización & Impuestos \\
\hline Ingreso a nuevos & Inseguridad Jurídica y \\
\hline nichos de mercado & Política \\
\hline Incentivos del & Cambios adversos en \\
\hline & Competencia desleal \\
\hline & Avances tecnológicos \\
\hline
\end{tabular}

Fuente: Investigación de Campo

Elaborado por: Autores 
En el FODA que antecede de las Pymes de Riobamba se evidencia que sus principales fortalezas son: prestigio, precios, personal calificado, a más de contar con diversificación de sus productos acorde a las exigencias del mercado consumidor, lo que les ha permitido proteger sus organizaciones ante la competencia. Sus principales debilidades son no tener una estructura organizacional definida, sistemas de control interno y de calidad no adecuados, y una debilidad que está en progreso, es la escasez de recursos económicos, lo que no ha permitido aumentar en gran medida los volúmenes de producción y mejorar su tecnología.

Las amenazas que los empresarios consideran transcendentes para su crecimiento son los impuestos, inseguridad económica, política y jurídica, que nos les permite avizorar un futuro prometedor, lo que incide en la decisión de arriesgarse a invertir en infraestructura o tecnología.

A pesar que el gobierno ha tomado medidas en beneficio de las PYMES; como en el caso de BanEcuador que otorga créditos de 5.000 a 500.000 dólares, con una frecuencia de pago personalizado de acuerdo al flujo de caja y ciclo productivo de cada proyecto; los empresarios consideran que no es oportuno endeudarse.

\section{Resultados de las Encuestas}

1. Su empresa cuenta con una guía que permita realizar un análisis de gestión administrativa financiera en el momento que usted lo considere oportuno.

Tabla 2. Empresas que cuentan con guía para su gestión Administrativa - Financiera

\begin{tabular}{lc}
\hline \multicolumn{1}{c}{ RESPUESTAS } & $\begin{array}{c}\mathbf{N}^{\circ} \text { de } \\
\text { Encuestados }\end{array}$ \\
\hline SI & 31 \\
NO & 29 \\
TOTAL & 60 \\
\hline
\end{tabular}

Fuente: Investigación de Campo

Elaborado por: Autores

En la encuesta realizada el $52 \%$ de los empresarios respondieron de forma afirmativa, expresando que sus organizaciones si poseen una guía, que les permite realizar un análisis de su gestión administrativa y financiera, la misma que admite una toma de decisiones oportuna, eficiente a más de minimización de riesgos; frente al $48 \%$ restante que manifiesta que en su empresa, la gestión administrativa y financiera la realizan de una forma empírica en base a la experiencia adquirida a través de los años de sus vivencias en el mercado.

2. ¿Al término de un ejercicio fiscal su organización realiza un análisis situacional de sus debilidades y fortalezas tanto Administrativas como Financieras?

Los empresarios encuestados respondieron en un $75 \%$, que al término de un ejercicio fiscal sus empresas realizan un diagnóstico situacional tanto administrativo como financiero, para 
determinar sus fortalezas y debilidades la misma que permite la toma decisiones oportunas, o los respectivos correctivos de ser necesario; frente a un $25 \%$ que no lo hace, por falta de tiempo en algunas ocasiones o por no tener las herramientas necesarias para hacerlo, tal como se muestra en la Tabla 3

Tabla 3. Diagnóstico situacional

\begin{tabular}{ll}
\hline RESPUESTAS & $\begin{array}{c}\mathbf{N}^{\circ} \text { de } \\
\text { Encuestados }\end{array}$ \\
\hline SI & 45 \\
NO & 15 \\
TOTAL & 60 \\
\hline
\end{tabular}

Fuente: Investigación de Campo

Elaborado por: Autores

3. ¿Su organización efectúa las actividades previas a una planificación?

Tabla 4. La Empresa Efectúa Planificación

\begin{tabular}{ll}
\hline \multicolumn{1}{c}{ RESPUESTAS } & \multicolumn{1}{c}{$\begin{array}{c}\mathbf{N}^{\circ} \text { de } \\
\text { Encuestados }\end{array}$} \\
\hline Siempre & 35 \\
A veces & 18 \\
Nunca & 7 \\
TOTAL & 60 \\
\hline
\end{tabular}

Fuente: Investigación de Campo

Elaborado por: Autores

El 58\% de las empresas que corresponde a 35 empresas, realizan una planificación de actividades previa a desarrollar sus diligencias administrativas y financieras, el $30 \%$ respondieron que realizan a veces una planificación que les permite conocer los recursos humanos, técnicos y económicos con los que cuentan, frente a un $12 \%$ que nunca lo realiza ya que toman decisiones de acuerdo como se presenten las diferentes situaciones en la empresa.

4. ¿La aplicación de prácticas Administrativas y Financieras aportarán a la sustentabilidad y sostenibilidad de su organización? 
Tabla 5. Aplicación de prácticas administrativas y financieras

\begin{tabular}{ll}
\multicolumn{1}{c}{ RESPUESTAS } & $\mathbf{N}^{\circ}$ de Encuestados \\
\hline SI & 44 \\
NO & 16 \\
TOTAL & 60 \\
\hline
\end{tabular}

Fuente: Investigación de Campo

Elaborado por: Autores

El 77\% de las empresas consideran que la aplicación de prácticas administrativas y financieras contribuyen en la sostenibilidad de las empresas y el $23 \%$ consideran que no son necesarias y que la experiencia les permite tomar decisiones, tal como se muestra en la tabla $\mathrm{N}^{\circ} 5$

5. ¿Cuenta su Empresa con estrategias para captar potenciales clientes en el sector?

El 73\% considera que en los actuales momentos, donde los mercados cada vez son más competitivos existe la necesidad apremiante de contar siempre con estrategias acordes a los avances tecnológicos que permitan captar potenciales clientes, y el $27 \%$ estima que su prestigio, productos o servicios son suficientes para mantenerse en el mercado.

Tabla 6. Estrategias Potenciar Clientes

\begin{tabular}{lc}
\hline \multicolumn{1}{r}{ RESPUESTAS } & $\mathbf{N}^{\circ}$ de Encuestados \\
\hline SI & 26 \\
NO & 34 \\
TOTAL & 60 \\
\hline
\end{tabular}

Fuente: Investigación de Campo

Elaborado por: Autores

El 73\% considera que en los actuales momentos, donde los mercados cada vez son más competitivos existe la necesidad apremiante de contar siempre con estrategias acordes a los avances tecnológicos que permitan captar potenciales clientes, y el $27 \%$ estima que su prestigio, productos o servicios son suficientes para mantenerse en el mercado.

6. ¿Su empresa aplica indicadores de gestión para evaluar los procesos?

Tabla 7. Aplica Indicadores

\begin{tabular}{cc}
\hline RESPUESTAS & $\begin{array}{c}\mathbf{N}^{\circ} \text { de } \\
\text { Encuestados }\end{array}$ \\
\hline SI & 46 \\
NO & 14 \\
TOTAL & 60 \\
\hline
\end{tabular}

Fuente: Investigación de Campo

Elaborado por: Autores 
7. ¿Su Empresa cuenta con un orgánico funcional que viabiliza la eficiencia administrativa?

Tabla 8. Orgánico Funcional

\begin{tabular}{ll}
\hline \multicolumn{1}{c}{ RESPUESTAS } & $\begin{array}{c}\mathbf{N}^{\circ} \text { de } \\
\text { Encuestados }\end{array}$ \\
\hline SI & 25 \\
NO & 35 \\
TOTAL & 60 \\
\hline
\end{tabular}

Fuente: Investigación de Campo

Elaborado por: Autores

El 58\% de los empresarios encuestados expresan que la empresa tiene un orgánico funcional, porque consideran que es necesario para dar la pauta de organización y orden jerárquico, el $42 \%$ manifiesta que no tiene elaborado un orgánico funcional, porque no lo han considerado importante para el desarrollo de sus actividades organizacionales.

¿La Aplicación de un Manual Administrativo Financiero optimizaría la gestión de su empresa?

El 75\% considera que un manual de gestión, daría lugar a un mejoramiento en las prácticas administrativas y financieras que permitan el cumplimiento de los objetivos planteados en sus empresas. Y el 25\% no lo considera indispensable.

Tabla 9. Aplicación Manual Administrativo- Financiero

\begin{tabular}{ll}
\hline & \multicolumn{1}{c}{$\mathbf{N}^{\circ}$ de } \\
EESPUESTAS & Encuestados \\
\hline SI & 45 \\
NO & 15 \\
TOTAL & 60 \\
\hline
\end{tabular}

Fuente: Investigación de Campo

Elaborado por: Autores

8. ¿La aplicación de indicadores de gestión contribuye a la toma decisiones eficaces?

Tabla 10. Aplicación de Indicadores de gestión

\begin{tabular}{|c|c|}
\hline RESPUESTAS & $\begin{array}{c}\mathbf{N}^{\circ} \text { de } \\
\text { Encuestados }\end{array}$ \\
\hline SI & 49 \\
\hline NO & 11 \\
\hline TOTAL & 60 \\
\hline
\end{tabular}

Fuente: Investigación de Campo

Elaborado por: Autores 
El $82 \%$ considera que el conocimiento y aplicación de indicadores de gestión permite tomar decisiones oportunas y eficaces en su empresa y el $18 \%$ no lo considera indispensable.

9. ¿Qué limitación considera usted relevante en la implementación de técnicas Administrativa y Financiera en su empresa?

El 37\% de los encuestados considera que la limitación más relevante para la implantación de técnicas administrativas y financieras, son los recursos, el $28 \%$ considera que es la falta de conocimientos, el $22 \%$ el desinterés y el 13\% el tiempo, tal como se muestra en la Tabla 11.

Tabla 11. Limitaciones en la implementación de Prácticas administrativas y financieras

\begin{tabular}{lc}
\hline \multicolumn{1}{c}{ RESPUESTAS } & $\begin{array}{c}\mathbf{N}^{\circ} \text { de } \\
\text { Encuestados }\end{array}$ \\
\hline Tiempo & 22 \\
Recursos & 17 \\
Falta de conocimientos & 8 \\
Desinterés & 13 \\
TOTAL & 60 \\
\hline
\end{tabular}

Fuente: Investigación de Campo

Elaborado por: Autores

10. ¿Indique a qué tipo de empresa pertenece?

Tabla 12. Tipo de Empresa

\begin{tabular}{lc}
\hline RESPUESTAS & $\begin{array}{c}\mathbf{N}^{\circ} \text { de } \\
\text { Encuestados }\end{array}$ \\
\hline Pequeña empresa & 47 \\
Mediana empresa & 13 \\
TOTAL & 60 \\
\hline
\end{tabular}

Fuente: Investigación de Campo

Elaborado por: Autores

El $22 \%$ es mediana empresa, frente al $78 \%$ es pequeña empresa; de acuerdo al análisis de las encuestas realizadas a los empresarios de la ciudad de Riobamba; en donde se pudo evidenciar que existe la necesidad de plantear un manual administrativo financiero que contribuya a mejorar las prácticas administrativas y financieras de las Pymes de Riobamba atendiendo a sus particularidades organizacionales.

4. Esquema de un Manual Administrativo y Financiero para una Pymes como una práctica para mejorar la gestión. 
1.Aspectos Generales de la Pymes

a. Nombre de la Organización

b. Dirección, Página Web, e-mail, teléfono

c. Marco Legal (Descripción de tipo de organización Jurídica, Acta de Constitución)

d. Accionistas u Socios de la Organización (Breve descripción de ser necesario)

2.Actividad de la Organización (Se describirá el tipo de actividad que realiza la organización)

3.Objetivos:
a. General
b. Específicos (Máximo tres relevantes)

4.Resumen Ejecutivo (No debe sobre pasar de dos hojas; se describirán los aspectos más relevantes de la organización)

5.Estructura de la Organización (Representar en forma sintética la organización)

a. Organigrama Estructural

b. Organigrama Funcional

c. Distribución de Funciones y Responsabilidades

d. Tiempo de Funcionamiento de los cargos

6.Recursos disponibles de la Organización
a. Económicos
b. Físicos
c. Equipamiento
d. Servicios Básicos

7.Planificación Administrativa
a. Misión
b. Visión
c. Valores Corporativos
d. Diagnóstico Situacional de la Organización
e. Matriz FODA
f. Matriz MEFI
g. Matriz MEFE
h. Determinación de Niveles de Riesgo 
i. Estrategias

j. Estrategias MEFI

k. Estrategias MEFE

\section{Planificación Financiera}

a. Plan de Cuentas

b. Estructura de los Estados Financieros

c. Formatos de Ingresos y Gastos

d. Plan de inversiones, clasificación y fuentes de financiamiento

e. Resumen de costos y gastos

f. Capital de trabajo

g. Flujo de caja

h. Detalle de las proyecciones de ingresos (ventas proyectadas)

i. Detalle de Indicadores

j. Punto de Equilibrio

k. Análisis de Costo Beneficio

Conclusiones:

- Se evidencia en la investigación realizada, que el 52\% de las Pymes encuestadas asegura poseer una guía que les permita realizar un análisis de gestión administrativa financiera evidenciando la importancia de utilizar herramientas de planificación para una acertada gestión organizacional.

- El 58\% de las empresas realizan planificación para optimizar el uso de sus recursos humanos, materiales y técnicos tendientes a buscar la sostenibilidad de las mismas. Existe la necesidad en el $73 \%$ de las Pymes encuestadas de contar con estrategias acorde a captar potenciales clientes en un contexto cada vez más competitivo en el que es necesaria la mejora continua.

- El 85\% considera que el conocimiento y aplicación de indicadores de gestión le permitiría una acertada toma de decisiones de forma técnica, sin empirismos, que contribuyan a la sostenibilidad de sus organizaciones y a la vez redefinir políticas que les consientan ser competitivas, en esta época donde la globalización de la economía obliga a un cambio apresurado de sus formas de organización empresarial, acorde a los avances que requieren los mercados actúales; más aún cuando sabemos que las pymes a nivel mundial contribuyen en gran medida a la dinamización de la economía 
y al desarrollo de las naciones. Lo que hace imprescindible que se adopte medidas estratégicas que permitan potenciar a este importante sector.

- Según lo expuesto podemos acotar que se hace imprescindible que se mejore las prácticas administrativas y financieras de las Pymes de Riobamba, ya que, según el desarrollo en los últimos tiempos de las ciencias administrativas, podemos encontrar un sin números de técnicas que contribuirían de forma efectiva en la gestión administrativa y financiera de las empresas.

- Una de las técnicas que se podría aplicar, es un Manual Administrativo y Financiero que serviría de instrumento de información, organizacional de funciones y control financiero, etc. Para un correcto desempeño en las organizaciones, tanto a nivel ejecutivo, encaminar a una buena dirección y al nivel operativo orientar el trabajo y sistematizar los procesos que permitan la mejora continua.

\section{EFERENCIAS BIBLIOGRÁFICAS}

- Anzola Rojas, S. (2002). Administración de pequeñas empresas. México: McGraw-Hill.

- Beltrán Jaramillo, J. M. (2000). Indicadores de Gestión. Herramientas para lograr la competitividad. 3R Editores.

- La Comisión de la Comunidad Andina. (9-10 de diciembre de 2008). Sistema Andino de Estadística de la PYME. Decisión 702. Lima, Perú.

- Luna, J. E. (2013). eumed.net. Obtenido de http://www.eumed.net/tesisdoctorales/2013/jelc/index.htm

- Martínez, G. (6 de Julio de 2016). Democraciaparticipativa.net.

- Stoner, J., Freeman, E., \& Gilbert, D. (1996). Administración. México D.F.: Prentice Hall.

- Goldman, D. (19 de Septiembre de 2012). Inventor. Obtenido de http://www.innmentor.com/2012/09/19/que-es-un-modelo/

- Muñiz, L. (2003). Cómo implementar un Sistema de Control de Gestión en la Práctica. Colombia: Instituto Colobiano de Normas Ténicas y Certificación ICONTEC.

- http://www.sri.gob.ec/web/guest/32 recuperado el 22 de agosto de 2017

- Amaru, A. (2009). Fundamentos de Administración (Primera ed.). México: Pearson Educación. 
- Stoner, J. (2000). Administración (Sexta ed.). México

- Tapia bonifaz , A. G. (2014). Tesis:Universidad Nacional de Chimborazo Riobamba.

- Muñiz, L. (2004). El diseño de un sistema de control de gestión. España: Ediciones Deusto.

- Córdoba Padilla, M. (2012). Gestión Financiera. Bogotá: Ecoe Ediciones.

- Greco, O. (2003). Diccionario de economía. (2a.ed.). Buenos Aires: Valletta Ediciones.

- Cipriano, L. G. A. (2016). Plan estratégico de negocios. Retrieved from https://ebookcentral.proquest.com 


\section{PARA CITAR EL ARTÍCULO INDEXADO.}

Tapia Bonifaz, A., Jácome Tamayo, S., Gavilánez Vega, M., \& Balseca Castro, J. (2019). Estudio de prácticas administrativas financieras en PYMES de Riobamba y su impacto en la gestión. Ciencia Digital, 3(3.2), 122-138. https://doi.org/10.33262/cienciadigital.v3i3.2.720

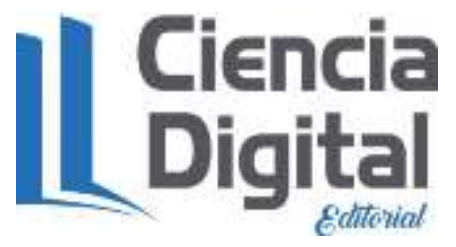

El artículo que se publica es de exclusiva responsabilidad de los autores y no necesariamente reflejan el pensamiento de la Revista Ciencia Digital.

El artículo queda en propiedad de la revista y, por tanto, su publicación parcial y/o total en otro medio tiene que ser autorizado por el director de la Revista Ciencia Digital.
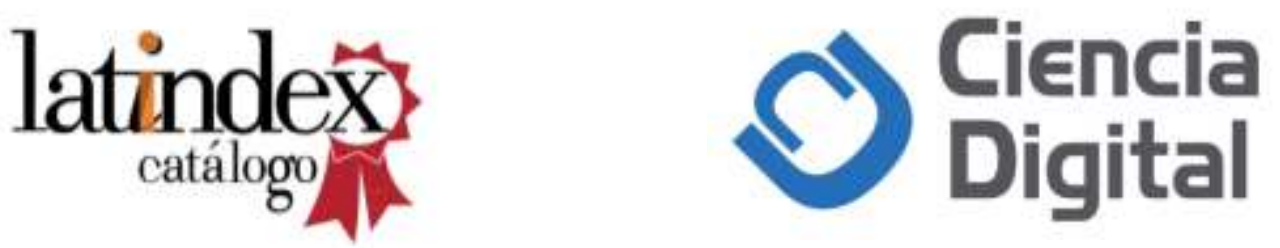